\title{
Part 1: Abstracts from the NCCN 19th Annual Conference: Advancing the Standard of Cancer Care ${ }^{\mathrm{TM}}$
}

The following abstracts were accepted for presentation at the NCCN 19th Annual Conference: Advancing the Standard of Cancer Care $^{\mathrm{TM}}$ General Poster Session at the Westin Diplomat in Hollywood, Florida on March 13-14, 2014. NCCN considered original abstracts from investigators in the oncology community related to Best Practices in Implementation and Use of Clinical Practice Guidelines, Quality Improvement, Outcomes and Health Services Research, and NCCN Oncology Research Program (ORP)-funded Projects.

Outcomes and Health Services Research

AB2014-1. Associated Between FDA Approval of Oncology Drugs and Relative Survival Benefits: An Analysis of the Oncology Drug Applications Over 10 Years

Idoroenyi Amanam, MD; Nichole Young-Lin, BA; Tuyen K. Kiet, BS; Kevin Blansit, BS; and John K. Chan, MD

Division of Gynecologic Oncology, UCSF Helen Diller Family Comprehensive Cancer Center

Background: Given that various cancers have different survival outcomes and varying levels of unmet need, the end points of hazard ratio, response rate, and progression-free and overall survivals may not be sufficient to predict for FDA approval of novel drugs. The authors evaluated relative survival benefit (additional benefit/ historic control) as an end point to predict for oncology drug approval.

Methods: Presentations and transcripts from Oncologic Drugs Advisory Committee (ODAC) sessions for drug license applications were reviewed. Relative survival benefit is defined as the percent improvement in survival associated with new therapy over standard therapy. The $\chi^{2}$ test and logistical regression were used in SPSS. Results: Of 23 drug license applications reviewed from 2001 to 2012, 12 were approved by the FDA. These drug applications comprised 5 drugs for breast cancer, 3 for pancreatic cancer, 2 for renal cancer, 2 for prostate cancer, 2 for soft tissue sarcoma, 2 for lung cancer, 2 for ovarian cancer, 2 for bone metastasis, 2 for brain cancer, and 1 for skin cancer (Table 1). A total of $83 \%$ studies used only overall survival (OS) as a primary end point, whereas the remainder used progression-free survival (PFS).

In all 24 studies, the median relative benefit was $10 \%$ (range, 3\%-120\%). Of the studies that used PFS $(n=4)$ versus $O S(n=20)$ as an end point, the average relative benefit was 90\% (range, 40\%-120\%) and 10\% (range, $0 \%-20 \%)(P=.02)$. Using the relative survival benefit of $10 \%$, those with higher relative survival benefit had an approval rate of $61 \%$ versus $20 \%$ in those with lower relative survival benefit, although this difference was not statistically significant $(P=.10)$. On subset analysis of studies using $\mathrm{OS}$ as the primary end point, no difference was seen in relative survival benefit $(P=.22)$. In a trends analysis of the relative survival benefit of the years 2001-2004, 2005-2008, and 2009-2012, no change was seen in the proportion of relative survival benefit over time $(P=.25)$.

Conclusions: The use of relative benefit may serve as a predictive indicator for prediction of FDA approval. Additionally, the relative benefit of drugs has not significantly improved. Clinical researchers need to consider these recommendations to improve the success of FDA approval of novel agents.

\begin{tabular}{|lc|}
\hline AB2014-1. Table 1: Types of Drug Applications \\
\hline Breast & 5 \\
Pancreatic & 3 \\
Renal & 2 \\
Prostate & 2 \\
Soft tissue sarcoma & 2 \\
Lung & 2 \\
Ovarian & 2 \\
Bone metastasis & 2 \\
Brain & 2 \\
Skin & 1 \\
Total & 23 \\
\hline
\end{tabular}

\section{AB2014-2. Novel Therapeutical Agents in Oncology: An Economic Point of View}

Carlos Camps, MD, PhD; ; Luis Paz-Ares, MD, PhD ${ }^{\text {b; }}$ Manuel Codes, MD, PhDc; Rafael López, MD, PhDd; Pere Gascón, MD, PhDe; Juan Jesús Cruz, MD, PhD;; Alfredo Carrato, MD, PhD;; Jesús García-Foncillas, MD, $\mathrm{PhD}^{\text {h; }}$ Vicente Guillem, MD, PhDi; and Eduardo Díaz-Rubio, MD, PhDj a Valencia University, 'Virgen del Rocío Hospital, 'Virgen Macarena University Hospital, 'Clinical University Hospital de Santiago de Compostela, ${ }^{e}$ Clinical University Hospital de Barcelona, ${ }^{f}$ University Hospital de Salamanca, '9Ramón y Cajal University Hospital, hJiménez Diaz Foundation, 'Valencian Institute of Oncology Foundation, 'San Carlos Clinical Hospital 
Background: The economic situation has shown that the resources devoted to health spending are limited, making rationalization of their consumption necessary. The relevance of pharmacoeconomic analyses is becoming crucial. The ECO Foundation, with the goal of promoting the quality of oncology care, set out to analyze the degree of consensus on the inclusion of new therapeutic targets and the integration of pharmacoeconomics when evaluating the effectiveness of these targets.

Methods: The study was conducted during the first ECO Seminar in 2010, using a modified Delphi method. An expert group discussed questions about pharmacoeconomic estimations, and analyzed the degree of consensus reached. The project was developed in 4 stages: 1) formation of the coordinating committee, 2) establishment of the panel of experts, 3) preparation and submission of the proposed conclusions of the seminar (e-mail survey in 2 rounds), and 4) analysis of the results and preparation of the agreed upon conclusions. Results: A total of 35 experts participated. With respect to the annual tolerable cost for the approval of new drugs, $68.8 \%$ considered a cost per life-year-gained between $€ 30,000$ and $€ 100,000$ acceptable, and 9.3\% found costs higher than $€ 150,000$ acceptable. With respect to the benefit of approving new drugs, for tumors with a median survival of less than 2 years, the median value that was considered clinically relevant was an increase of 3 months, either in the time to progression (TTP) or the overall survival (OS). In neoplasms with more than 2 years mean survival, an increase of 3 to 4 months in TTP and of 4 to 6 months in OS was considered clinically relevant. By consensus, standard chemotherapy was considered to be effective if it produced an improvement in median survival of at least 3 to 4 months in at least $20 \%$ of the patients treated.

Conclusions: The costs of new drugs are higher than those of traditional treatments, making it a priority to identify subgroups of patients with specific molecular profiles who are candidates for higher-efficiency targeted therapies. Allocation of available resources to the most effective interventions, to achieve the best clinical outcomes with lower costs and best subjective profile possible, allows expenditure to be rationalized. Pharmacoeconomic studies are a basic tool for obtaining better health outcomes based on the available resources, while also considering the other needs of the population.

\section{AB2014-3. Understanding the Patient Experience of ALK+ Non-Small Cell Lung Cancer \\ Jennifer Devlen, $\mathrm{PhD}^{\mathrm{a}}$; Medha Sasane, $\mathrm{PhD}^{\mathrm{b}}$; \\ Amanda Doyle, MA, CCRP; Emuella Flood, BAa: \\ Nada Saleh, MA; ${ }^{\mathrm{a}}$ Monique Monita, $\mathrm{MPH}^{\mathrm{b}}$; and Ken Culver, $\mathrm{MD}^{\mathrm{b}}$ \\ a/CON Commercialisation \& Outcomes; ${ }^{b}$ Novartis Pharmaceuticals Corporation; 'Highlands Oncology Group}

Background: Patients with anaplastic lymphoma kinase-positive $(\mathrm{ALK}+)$ non-small cell lung cancer (NSCLC) are rare, representing approximately 5\% of all NSCLC cases. The burden of illness from the patient perspective is not well explored and a review of literature failed to identify any qualitative studies in this population. This qualitative study aimed to describe the symptoms and impacts of ALK+ NSCLC from the patient's perspective.

Methods: In depth one-on-one interviews were conducted with adults with ALK+ NSCLC. Using a standardized interview guide, a trained interviewer asked open-ended questions to elicit patients' experiences of diagnosis, symptoms, impacts, and treatment. Interviews were audio-recorded and transcribed, and a thematic analysis was conducted to identify key concepts using qualitative software (MaxQDA). In addition, a review of Internet blogs and patient forums was conducted.

Results: Preliminary findings from 12 interviews (mean age, 56.8 years; SD, 8.6; 9 women) and 6 blogs revealed varying experiences. At diagnosis, some patients reported lung symptoms such as dyspnea, cough, wheezing, hoarseness, and chest discomfort that made it difficult to speak, walk, climb stairs, or sleep, and interfered with work and household activities. Others presented with no symptoms and indicated that lung cancer was an accidental finding during other health-related investigations. The diagnosis of lung cancer was sometimes delayed because of nonsmoking status and younger age. Although some patients expressed despair and the shock of being diagnosed with NSCLC, others emphasized positive aspects of being ALK+ because of better treatment options and being able to avoid debilitating and intolerable effects of chemotherapy. Most patients felt stigma because of its association with smoking and could not identify with the larger community of patients with lung cancer. Those diagnosed with early-stage disease received chemotherapy and crizotinib on recurrence once ALK+ status was identified. Patients diagnosed more recently were tested for ALK fusion at initial diagnosis and were treated with crizotinib. Most patients had switched treatment because of disease progression or treatment toxicity. With progression, patients report- 
ed reappearance of symptoms. Those with metastases to brain reported dizziness or loss of hearing, and those with metastasis to bone reported pain or fractures that impacted daily activities.

Conclusions: The diagnosis of ALK+ NSCLC is unexpected, devastating, stigmatizing, and often delayed, because these patients tend to be younger and nonsmoking, and have wide-ranging symptoms. Patients report significant impacts on daily activities at initial diagnosis and at progression, including inability to work. Patients who are ALK+ perceive themselves to be different from the larger NSCLC community. They believe they have better treatment options and are generally hopeful about new treatments on the horizon.

\section{Clinical Oncology Research Trials}

\section{AB2014-4. Uridine Triacetate: Antidote for}

\section{5-Fluorouracil Overexposure}

Michael Bamat, PhD; Robert Tremmel, PharmD;

and Reid von Borstel, PhD

Wellstat Therapeutics Corporation

Background: 5-Fluorouracil (5-FU) is broadly used to treat solid tumors. It is typically administered via intravenous infusion, at or near its maximum tolerated dose, over several days. Life-threatening or lethal 5-FU toxicity occurs because of errors in programming infusion pumps, infusion reservoir errors, and dosage miscalculations. Patients with dihydropyrimidine dehydrogenase (DPD) deficiency (up to $3 \%$ of the population), which leads to impaired 5-FU elimination, also experience serious or lethal toxicity. In addition, some patients experience unusual, severe, early-onset toxicities from exposure to 5-FU, such as encephalopathy or cardiotoxicity, including cardiac arrest.

Uridine triacetate is an orally bioavailable prodrug of uridine, the direct biochemical antagonist of 5-FU. Uridine triacetate has been used successfully to treat patients in emergency 5-FU overdose situations, patients with known or suspected overexposure from DPD deficiency, and patients with 5-FU-induced, sudden-onset neurotoxicity and cardiotoxicity.

More than 125 patients at excess risk of 5-FU toxicity from overdose, accidental capecitabine ingestion, or possible DPD deficiency, or who displayed rapid onset of severe toxicities, have been treated with uridine triacetate using a common treatment regimen and protocol. Methods: Uridine triacetate was provided under Emergency Investigational New Drug provisions or an expanded access protocol when requested by qualified clinical sites after 5-FU overexposures (most as a result of infusion pump errors) or early onset of severe toxicities. Patients received uridine triacetate $(10 \mathrm{~g}$ every 6 hours for 20 doses) as soon as possible after recognition of the overdose or possible clearance defect. Clinical outcomes were monitored, including safety, survival, and resumption of chemotherapy.

Results: To date, 125 patients who have experienced overexposure to 5-FU have been treated with uridine triacetate. Of these, 122 recovered fully. Reductions in or absence of anticipated gastrointestinal, hematologic, and other toxicities associated with 5 -FU poisoning were seen, and rapid reversal of 5-FU-induced encephalopathy and myocardial dysfunction were observed. Mild or no adverse events were attributed to uridine triacetate.

Conclusions: Uridine triacetate seems to be a safe and effective life-saving antidote for 5-FU overexposure in emergency situations.

\section{Human Subject Research Protection \\ AB2014-5. NCCN's Informed Consent Language Database \\ Emily Eldh, CIPa; Kristofer Griffith, CIPb; Jennifer Hackworth ${ }^{\text {b; }}$ Donald Handley, MSc, MBA, CIPc; Jan Hewett, BSN, JDd; Diane Paul, MS, RNe; and Donna Scharffe

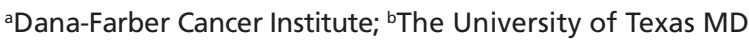 Anderson Cancer Center; 'Roswell Park Cancer Institute; ${ }^{\text {YYale }}$

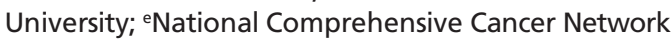

Background: The consent process is a cornerstone of human subject research protection. Informed consent documents are required by federal regulations. Specific elements of informed consents need to be addressed, as outlined in the Belmont Report and federal regulations. One of the most important elements is that of comprehensibility. Review and revision of informed consents by Institutional Review Boards (IRBs) can add considerable time to the review process. The use of standardized language that is easily understood by human subjects can significantly reduce the time spent by IRBs on this aspect of approving clinical research. Having standardized template language for clarity and consistency can ensure that information is presented clearly.

Methods: The NCCN Informed Consent Language (ICL) database is a free comprehensive resource to assist researchers and clinical operations personnel with writing and amending informed consents for study participants. It currently contains more than 2200 standardized lay-language descriptions of procedures, risks, and events associated with clinical research. The database content was developed through collaboration with 2 NCCN Member Institutions, namely Dana-Far- 
ber Cancer Institute and The University of Texas MD Anderson Cancer Center. Both institutions' IRBs routinely review, approve, and update the standardized language to ensure consistency and accuracy. NCCN is committed to supporting initiatives to improve clinical research processes, and plans to expand the database to include language regarding mechanisms of action for drugs.

Results: Since the launch of the database on August 16, 2012, the database has more than 4200 unique visitors from 90 countries.

Conclusions: A user satisfaction survey is planned to understand the utility of the database and to query its effect on IRB review time.

\section{Quality Improvement}

AB2014-6. Staging Studies in Early-Stage Breast Cancer at a Community-Based County Hospital Before the Latest Recommendations From ASCO

Punprapai Boriboonsomsin, MD; Ta Vinhfield, MD; and Jeffrey Miller, MD

UCLA Olive View Medical Center

Background: "Five Things Physicians and Patients Should Question" published by ASCO in May 2012 recommended that PET, CT, and radionuclide bone scans not be performed in patients with early-stage breast cancer. These tests are often used in the staging evaluation of low-risk cancers despite a lack of evidence suggesting that they improve detection of metastatic disease or survival. Using data from their community-based county hospital, the authors determined the number of staging studies that were ordered for patients with stages I and II breast cancer before the recommendations were issued, and evaluated the effect of the staging studies on the time from diagnosis to receiving first treatment.

Methods: Using the electronic medical record system, the authors compiled data records of patients with newly diagnosed stages I and II breast cancer between 2008 and 2012. Data include whether CT chest, CT abdomen/pelvis, PET scan, bone scan, and brain imaging studies were ordered; reason for ordering them; and the time to first treatment with either neoadjuvant chemotherapy or surgery.

Results: At least one staging study was ordered for $41 \%$ (92 of 224) of the patient samples, of which $76 \%$ (70 of 92) had no record of the reason for ordering the studies (Table 1). None of the patient samples who had at least one staging study ordered were upstaged. On average, the time to first treatment was longer for the patient samples with at least one staging study than for those without (49 vs 42 days; $P=.04$ ).

Conclusions: In the past 5 years, $31 \%$ of the patients with newly diagnosed stages I and II breast cancer at the authors' hospital (70 of 224) were ordered staging studies without a recorded reason, which caused a delay in receiving the first treatment by an average of 1 week. This can be improved through following the recommendations regarding staging studies published by ASCO in May 2012.

AB2014-6. Table 1: Studies Ordered for

Patients With Stages I and II Breast Cancer

Between 2008 and 2012

\begin{tabular}{|c|c|c|c|c|}
\hline Stage & 1 & IIA & IIB & Total \\
\hline N & 92 & 75 & 57 & 224 \\
\hline СT Chest & 12 & 31 & 33 & 76 \\
\hline $\begin{array}{l}\text { CT Abdomen/ } \\
\text { Pelvis }\end{array}$ & 12 & 29 & 35 & 76 \\
\hline PET Scan & 12 & 22 & 34 & 68 \\
\hline Bone Scan & 1 & 8 & 4 & 13 \\
\hline Brain Imaging & 4 & 5 & 6 & 15 \\
\hline $\begin{array}{l}\text { Patients With } \\
\text { Orders [\%] }\end{array}$ & $18(20 \%)$ & $34(45 \%)$ & $40(70 \%)$ & $92(41 \%)$ \\
\hline $\begin{array}{l}\text { Reason Not } \\
\text { Recorded [\%] }\end{array}$ & $10(56 \%)$ & $26(76 \%)$ & $34(85 \%)$ & $70(76 \%)$ \\
\hline Upstaged & 0 & 0 & 0 & 0 \\
\hline
\end{tabular}

AB2014-7. Chemotherapy and Bone Marrow Transplant Mobilization and Optimal Harvest Time

Jeanine Chiaffarano, DO; Rebecca Gudgin, BS; and

Mercy Kuriyan, MD, MHA

Rutgers Robert Wood Johnson Medical School

Background: Various types of chemotherapy are used in hematologic malignancies. Patients who are potential hematopoietic stem cell transplant recipients are mobilized for harvest using chemotherapy effective for the type of malignancy. The time to mobilization can vary depending on the therapy. The ability to predict the best date to initiate harvest helps in treatment planning for the patient and the collection facility. Optimal timing of harvest also helps decrease the number of collections and increase the dose derived per collection. To assess the optimal date for harvest by disease and therapy, a retrospective study was performed of patients who were mobilized with chemotherapy.

Methods: Patients $(n=111)$ in the database for a 10 year period (2002-2012) who were mobilized with chemotherapy were evaluated for diagnosis, type of chemotherapy (Table 1), days to first collection, and number of collections. Average and range by disease and chemotherapy were derived for days to first collection and number of collections. 
Results: For non-Hodgkin's lymphoma $(n=54)$, the average days to first collection/average number of collections were 11.0/1.5 for RCHOP; 13.0/1.25 for CHOP; 13.5/1.0 for BEAM; 14.0/1.0 for DHAP, RDHAP; $14.75 / 1.50$ for ICE; and 16.0/1.4 for RICE. For Hodgkin lymphoma $(n=31)$, the average days to first collection/average number of collections were 15.0/1.5 for ICE and 15.0/1.0 for RICE. For multiple myeloma $(n=22)$, the average days to first collection/average number of collections were 13.0/2.0 for cyclophosphamide; 15.0/1.5 for REPOCH; and 16.0/1.5 for EP$\mathrm{OCH}$. For germ cell tumors $(n=4)$, the average days to first collection/average number of collections were 13.0/3.25 for paclitaxel and ifosfamide.

Conclusions: Results showed that in patients with non-Hodgkin's lymphoma, when BEAM, DHAP, and RDHAP are used for mobilization, the optimal time to harvest is 2 weeks; for those with multiple myeloma, the average is 15 days; and for those with Hodgkin lymphoma, it is 15 days irrespective of therapy choice. For germ cell tumors, optimal time to collection requires further assessment. The time to harvest demonstrated in this study can be used to ensure adequate staffing and maximize collections.

\begin{tabular}{|ll|}
\hline AB2014-7. Table 1: Chemotherapeutic Regimens \\
CHOP & $\begin{array}{l}\text { Cyclophosphamide, hydroxydaunorubicin, } \\
\text { vincristine, prednisone }\end{array}$ \\
RCHOP & CHOP + rituximab \\
BEAM & $\begin{array}{l}\text { Carmustine (BiCNU), etoposide, cytarabine } \\
\text { (arabinoside), melphalan }\end{array}$ \\
DHAP & $\begin{array}{l}\text { Dexamethasone, cytarabine (Ara-C), } \\
\text { cisplatin (platinum) }\end{array}$ \\
RDHAP & DHAP + rituximab \\
ICE & Ifosfamide, carboplatin, etoposide \\
RICE & ICE + rituximab \\
EPOCH & Etoposide, prednisone, vincristine, \\
& cyclophosphamide, hydroxydaunorubicin \\
REPOCH & EPOCH + rituximab \\
\hline
\end{tabular}

\section{Cancer Survivorship \\ AB2014-8. CancerSupportSource (CSS): Validating a13-Item Web-Based Distress Screening Tool in the Community}

Joanne Buzaglo, PhD; Melissa F. Miller, PhD, MPH;

Christopher Gayer, PhD; Anne Morris, MPH;

Vicki Kennedy, LCSW; and Mitch Golant, PhD

Cancer Support Community Research and Training Institute

Background: The IOM, NCCN, and American College of Surgeons have recognized that screening for psychosocial concerns is critical to ensuring quality cancer care for the whole patient. Few tools have been validated in the community, where up to $85 \%$ of patients with cancer are treated. CancerSupportSource (CSS) is a 25-item Web-based tool (CSS-25) designed to screen patients for distress and connect them with appropriate resources. CSS-25 asks patients to rate their concerns on a scale of $\mathrm{O}$ (not at all) to 4 (very seriously concerned) and indicate whether they want to 1) talk with a health care team member; 2) receive print information; or 3) access online resources. The purpose of this study was to test the psychometric properties of a shorter 13-item version (CSS-13), including a 4-item depression subscale, among a community-based sample of cancer survivors.

Methods: A convenience sample of 251 English-speaking cancer survivors was recruited across 10 affiliates of a cancer support organization. Participants (90\% women, median age 57 years) completed a Web-based survey including CSS-25, the Functional Assessment of Cancer Therapy: General well-being scale (FACT-G), the Center for Epidemiologic Studies Depression Scale (CESD), and the Distress Thermometer (DT). The first 100 respondents completed CSS-25 a second time to measure test-retest reliability.

Results: CSS-13 demonstrated high internal reliability (Cronbach's alpha=0.91). Test-retest reliability was strong (intraclass correlation coefficient $[\mathrm{ICC}] \geq 0.75$ ) for 9 of the items and moderate for $4(0.65 \leq \mathrm{ICC}<0.75)$. The median distress score (sum of 13 item ratings) was 14 and correlated well with the FACT-G $\left(R^{2}=0.50\right.$; $P<.001)$, CES-D $\left(R^{2}=0.52 ; P<.001\right)$, and DT $\left(R^{2}=0.40\right.$; $P<.001)$, indicating moderate to strong concurrent validity. The correlation with "gold standard" measures was stronger for CSS-13 than for CSS-25. In receiver operating characteristic (ROC) analysis, a score of 14 or greater on the CSS-13 had a true-positive rate (sensitivity) of $95 \%$ and a false-positive rate (specificity) of $9 \%$ compared with a score of 25 or greater on the CSS- 25. In other words, 118 of the 124 (95\%) who indicated risk of distress on the CSS-25 would also be classified as at risk on the CSS-13. For the 4-item depression subscale (sad, lonely, nervous, fatigue; alpha=0.83), the sensitivity and specificity for depression (CES-D $\geq 21$; area under the curve $[A U C]=0.92$ ) was $97.5 \%$ and $72.2 \%$, respectively, if the depression score (sum of 4 problem ratings) was 5 or greater. The 13 -item scale was able to discriminate groups of clinical relevance (active treatment, time since diagnosis). The distress score was not different by stage of cancer at diagnosis. 


\section{AB2014-9. CancerSupportSource: Validating a 15-Item Spanish Web-Based Distress Screening Tool in the Community \\ Christopher Gayer, PhD; Melissa F. Miller, PhD, MPH; Anne Morris, MPH; Mitch Golant, PhD; Vicki Kennedy, LCSW; and Joanne Buzaglo, PhD \\ Cancer Support Community Research and Training Institute}

Background: The IOM, NCCN, and American College of Surgeons have recognized that screening for psychosocial concerns is critical to ensuring quality cancer care for the whole patient. Few tools have been validated for Spanish speakers in the community, where up to $85 \%$ of patients with cancer are treated. CancerSupportSource-Spanish is a 25-item Spanish Webbased tool (CSS-Sp-25) designed to screen patients for distress in the community and connect them to appropriate resources. CSS-Sp-25 asks patients to rate their concerns across 25 items using a 5 -point scale $(0=$ not at all; $4=$ very seriously concerned). For each item, patients indicate whether they want to 1) talk with a health care team member; 2) receive print information; or 3) access online resources. The purpose of this study was to test the psychometric properties of a shorter 15 -item version (CSS-Sp-15), including a 4-item depression subscale, among a community-based sample of cancer survivors.

Methods: A convenience sample of 182 Spanish-speaking cancer survivors was recruited across 6 affiliates of a cancer support organization. Participants (91\% female, median age, 54 years) completed a paper-and-pen survey including the CSS-Sp-25, the Functional Assessment of Cancer Therapy-General well-being scale (FACT-G), the Center for Epidemiologic Studies Depression Scale (CES-D), and the Distress Thermometer (DT).

Results: CSS-Sp-15 showed high internal reliability (Cronbach's alpha=0.94). The median distress score (sum of 15-item ratings) was 23, with $64 \%$ indicating scores at or above 17 , and correlated moderately with the FACT-G $\left(R^{2}=0.35 ; P<.001\right)$, CES-D $\left(R^{2}=0.46\right.$; $P<.001)$, and DT $\left(R^{2}=0.24 ; P<.001\right)$, indicating moderate concurrent validity. The correlation with "gold standard" measures was approximately equivalent for CSS-Sp-15 and CSS-Sp-25. In receiver operating characteristic analysis, a score of 17 or greater on the CSS15 has a true-positive rate (sensitivity) of $97 \%$ and a false-positive rate (1-specificity) of 5\% compared with a score of 28 or greater on the CSS-25 (area under the curve [AUC], 0.99). In other words, 113 of the 117 (95\%) of the respondents who indicated risk of distress on the CSS-Sp-25 would also be classified as at risk on the CSS-Sp-15. For the 4-item depression subscale (sad, lonely, nervous, fatigue; alpha=0.89), the sensitivity and specificity for depression (CES-D $\geq 21 ; A U C=0.85$ ) was $91.8 \%$ and $69.0 \%$, respectively, if the depression score (sum of 4 problem ratings) was 7 or more.

Conclusions: CSS-Sp-15 showed moderate to strong psychometric properties and can be a valuable instrument to screen for psychosocial distress. These results have important implications for the delivery of screening and psychosocial referral for underserved populations. Future research will test the implementation of CSS-Sp-15 for cultural sensitivity in diverse Spanishspeaking communities.

Conclusions: CSS-13 shows strong psychometric properties that can help screen for patients at high risk for distress and depression. Addressing the needs of these patients through appropriate screening, referral, and follow-up can lead to better patient-reported outcomes.

Gastrointestinal Stromal Tumors, Risk of Recurrence, Risk Assessment

AB2014-10. Underestimation of the Risk of Recurrence in Patients with Primary Resected Gastrointestinal Stromal Tumors

Anthony P. Conley, MDa; Medha Sasane, PhD ${ }^{\text {; }}$;

Annie Guerin, MSc; Alexander R. Macalalad, MD; Qing Huang, MHSc; Frances Schwiep, BSc; and Eric Wu, PhDc

${ }^{a} \mathrm{MD}$ Anderson Cancer Center; ${ }^{b}$ Novartis Pharmaceuticals Corporation; 'Analysis Group, Inc.

Background: After complete resection of a primary gastrointestinal stromal tumor (GIST), recurrence can be reduced with at least 3 years of adjuvant therapy, particularly among patients with GIST at high risk of recurrence. Several scoring systems have been developed to assess the risk of recurrence, but how physicians score this risk and how well they do is unclear. This study explored how physicians assess risk of recurrence, and how their assessment compares with the revised NIH criteria.

Methods: In this retrospective chart review, an online data collection form was completed by participating US oncologists on randomly selected adult patients diagnosed with a primary resectable GIST. For each patient, physicians reported their charted risk assessment and the clinical parameters needed to categorize risk under the revised NIH criteria. A weighted kappa statistic assessed the level of agreement between physicians and the revised $\mathrm{NIH}$ criteria, and a sign test assessed the difference. Logistic regressions assessed predictors of underestimated risk, adjusting for potential patient-and physician-level confounders.

Results: A total of 109 oncologists contributed 506 patients to the study (Table 1). On average, patients 
were 59 years of age (SD, 11$)$, male $(54.9 \%)$, and white (53.2\%). Primary tumors were mainly gastric $(42.3 \%)$ and greater than $5 \mathrm{~cm}$ in size $(51.6 \%)$, with a mitotic count greater than 5/50 high-power field (HPF; 68.1\%), and most underwent resection without rupture (81.8\%). Based on the revised NIH criteria, $65.8 \%$ of patients were at high risk of recurrence, $8.7 \%$ at intermediate risk, and $25.5 \%$ at low or very low risk. In comparison, the physicians' charted assessment underestimated risk for $37.5 \%$ of the patients $(P<.001$ sign test; weighted kappa $=0.53$ ). Physicians were more likely to underestimate risk for resected tumors 5.1 to $10.0 \mathrm{~cm}$ versus those $5.0 \mathrm{~cm}$ or less or greater than $10.0 \mathrm{~cm}(50.3 \%$ vs $30.7 \%$; $P<.001$ ); with amitotic count of $6 / 50$ to $10 / 50 \mathrm{HPF}$ versus $5 / 50 \mathrm{HPF}$ or less or greater than 10/50 HPF $(63.1 \%$ vs $21.5 \% ; P<.001)$; and nongastric versus gastric resected GISTs $(45.5 \%$ vs $26.6 \% ; P<.001)$. In multivariate analysis, predictors of underestimation included tumor size of 5.1 to $10.0 \mathrm{~cm}$ versus $5.0 \mathrm{~cm}$ or less (odds ratio, 1.93 ; $P=.034$ ); mitotic count of $6 / 50$ to $10 / 50 \mathrm{HPF}$ versus $5 / 50 \mathrm{HPF}$ or less (odds ratio, $6.64 ; P<.001$ ), and nongastric versus gastric GIST (odds ratio, 2.00; $P=.003$ ).

Conclusions: Physicians frequently underestimate the risk of GIST recurrence, particularly for resected GISTs 5.1 to $10.0 \mathrm{~cm}$, or with a mitotic count of $6 / 50$ to $10 / 50$ HPF, or a nongastric GIST. Further study is needed to determine the impact of this underestimation on rates of recurrence, especially in high-risk patients.

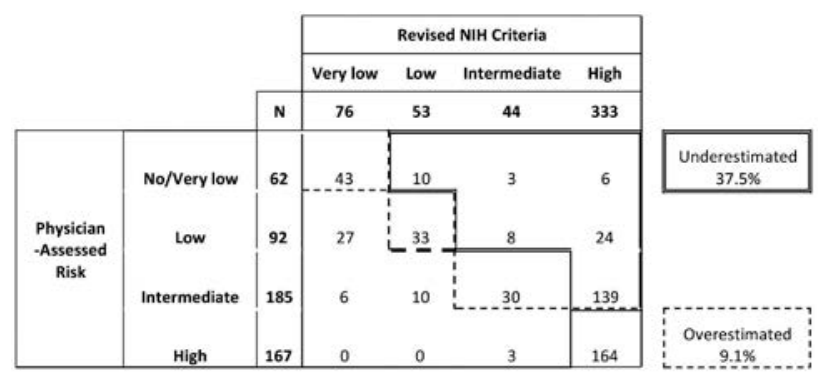

AB2014-10. Table 1: Comparison of Risk Categorization

\section{Bladder Cancer}

AB2014-11. Chronic Kidney Disease Epidemiology and Cockcroft-Gault Equations Identify Similar Candidates for Neoadjuvant Chemotherapy in Muscle-Invasive Bladder Cancer

Mark Chang, BSc ${ }^{\mathrm{a}}$; Nora Ruel, MA ${ }^{\mathrm{b}}$; Sergio Villegas, $\mathrm{BS}^{\mathrm{a}}$; Timothy G. Wilson, MD;; Nicholas J. Vogelzang, MD'; Bertram E. Yuh, MD; ; and Sumanta K. Pal, MDa

${ }^{a}$ Department of Medical Oncology \& Experimental Therapeutics, ${ }^{b}$ Division of Biostatistics, Department of Information Science, and 'Division of Urology, Department of Surgery, City of Hope Comprehensive Cancer Center; and ${ }^{d}$ US Oncology Research, Comprehensive Cancer Centers
Background: NCCN Clinical Practice Guidelines in Oncology (NCCN Guidelines) suggest strong consideration of neoadjuvant cisplatin-based chemotherapy before cystectomy in the setting of muscle-invasive bladder cancer (MIBC). A creatinine clearance $(\mathrm{CrCl})$ greater than $60 \mathrm{~mL} / \mathrm{min}$ is frequently used to characterize cisplatin-eligible patients and use of the chronic kidney disease epidemiology (CKD-EPI) equation to estimate $\mathrm{CrCl}$ has been advocated. The objective of the current study was to determine the proportion of patients determined to be cisplatin-eligible based on the CKD-EPI equation compared with the Cockroft-Gault (CG) equation and other conventional methods.

Methods: From a prospectively maintained institutional database, patients with MIBC who received cystectomy were identified and clinicopathologic information was ascertained. $\mathrm{CrCl}$ before surgery was computed using 3 equations: CG, CKD-EPI, and Modified Diet in Renal Disease (MDRD). The primary objective was to determine whether the CG and CKD-EPI equations identified a different proportion of patients who were cisplatin-eligible based on an estimated $\mathrm{CrCl}$ of greater than $60 \mathrm{~mL} / \mathrm{min}$. Cisplatin-eligibility was also assessed in subsets based on age, Charlson comorbidity index score, and race. Actuarial rates of neoadjuvant cisplatin-based chemotherapy use were also reported.

Results: Of 126 patients, $70 \%$ and $71 \%$ were found to be cisplatin-eligible using the CKD-EPI and CG equations, respectively $(P=.9)$. The MDRD did not result in significantly different characterization of cisplatin-eligibility compared with the CKD-EPI and $\mathrm{CG}$ equations. In the subset of patients older than 80 years, the CKD-EPI equation identified a much smaller proportion of those who were cisplatin-eligible $(25 \%)$ compared with the CG and MDRD equations (50\% and 63\%, respectively). Only 34 patients (27\%) received neoadjuvant cisplatin-based chemotherapy. Of the 92 patients who did not receive neoadjuvant chemotherapy, $64 \%$ had a $\mathrm{CrCl}$ greater than $60 \mathrm{~mL} / \mathrm{min}$ by CG.

Conclusions: In contrast to previous reports, the CKD-EPI equation does not seem to characterize a broader span of patients as cisplatin-eligible. Older patients (age $>80$ years) may less frequently be characterized as cisplatin-eligible by CKD-EPI. The discordance between actual rates of neoadjuvant chemotherapy use and rates of cisplatin eligibility suggest that other factors (eg, patient and physician preference) may guide clinical decision-making. 